\title{
Commentary
}

\section{The National Atmospheric Emissions Inventory System}

The South African air quality governance cycle (National Framework, 2012) illustrates that informed decision-making is fundamental to good governance, and decisions can only be properly informed if decision-makers have ready access to accurate, relevant, current and complete information. Thus, the information management component of the governance cycle is crucial and it can be defined as the engine that drives the cycle towards continuous improvements in environmental quality. In light of that, the Department of Environmental Affairs (DEA) has established the South African Air Quality Information System (SAAQIS), which is hosted by the South African Weather Service (SAWS). The information and knowledge management needs of government; as implied in national legislation are being met through the SAAQIS. The SAAQIS is divided into phases; the first being the ambient air quality module, which is also an information repository of all government air quality management documents. There are over a hundred monitoring stations in the country which report ambient air quality monitoring data to the SAAQIS and this enables all stakeholders, including government and the general public, to access verified data showing the state of air quality at any given point in time.

The same has not been the case, however, when it comes to atmospheric emissions data and reporting. Due to the lack of uniform reporting requirements in the past, very few significant sources of atmospheric emissions, like large industries, or holders of data that could be used in estimating significant sources of atmospheric emissions, such as the Department of Energy, were required to submit these data to the spheres of government (national, provincial and local) responsible for air quality management. Where reporting had been taking place, emissions reports were submitted to various national, provincial or municipal authorities. As a result, the national atmospheric emission data set is incomplete and the data collected are extremely limited. Thus, the collation of provincial and/or national emission data sets is complex, time-consuming and expensive, and may not even provide complete, accurate, current and/or relevant data sets due to the use of completely different methodologies for calculating or estimating emissions.

Further, the emissions reports themselves are either submitted in hard-copy (written or typed reports on paper) or submitted in different electronic formats such as text documents or spreadsheets. The result is that the data are difficult to capture in a way that would allow proper analysis and comparison between sources hence they cannot be used with any acceptable level of confidence. This means that the national atmospheric emission profile is difficult, if not impossible, to assess to any level of acceptable confidence; hence it is impossible to model the national 'state of the air'. Moreover, it is difficult, costly and time-consuming to estimate the potential impact of new developments on ambient air quality without the ability to model cumulative impacts. This makes environmental impact assessments insufficient to ensure that new developments do not have an unacceptable impact on the quality of ambient air since decision-makers cannot model the future impact of a development on ambient air quality.

It is evident in light of all the problems mentioned above, that South Africa needs a complete, verified and comparable national atmospheric emissions inventory. In this regard, and in-line with regulatory requirements, the National Air Quality Officer has established an internet-based National Atmospheric Emission Inventory System (NAEIS). At the time of writing this editorial, the Department is in the process of finalising the reporting regulations for the system. With the establishment of the NAEIS, all significant industrial emitters who have atmospheric emission licenses shall submit annual emissions inventory reports in the form necessary for the compilation of the national emission inventory profile. The system shall also provide an emission inventory reporting platform for all non-listed activities that produce atmospheric emissions. The atmospheric emissions will be estimated inside or outside the NAEIS depending on the emission sources types.

Further, the NAEIS will provide guidance and methodologies for the compilation of atmospheric emission inventories following best international practices, and these tools will be available for emission inventory compilation by all spheres of government. The NAEIS will also serve as a platform for current and future emissions estimations. The NAEIS, once fully operational, will include details of emissions from all source types (e.g. point, mobile, area, line, volume), details of emissions by pollutant type, norms and standards for emission inventories compilation, search tools to interrogate the inventories, database of emission factors for various activities; among others. The inventory of atmospheric emissions from all source sector types will be accessible from the NAEIS. Of note, the Department has received compliments from some developed countries for the NAEIS due to its vast capabilities as listed herein; indeed the achievement is a "leap-frog" in the area of atmospheric emissions reporting and databases.

Finally, a National Atmospheric Emission Inventory System Data Management Policy will be developed to specify the protocols for data management and levels of accessibility for all users, including stakeholders/general public. It was initially envisaged that the NAEIS would be developed as a component of SAAQIS as phase two; however, the data management policy and associated work packages will give guidance with regard to the specific location of the NAEIS according to legal mandates, objectives of and the desired integrity of the system. The successful implementation of the NAEIS will mean that South Africa has adequate information to begin considering real-time air quality reporting and forecasting.

\section{Dr Thuli Mdluli}

National Air Quality Officer, Climate Change and Air Quality Branch, Department of Environmental Affairs 